\title{
ON COUNTABLY CLOSED COMPLETE BOOLEAN ALGEBRAS
}

\author{
Thomas Jech and Saharon Shelah \\ The Pennsylvania State University \\ The Hebrew University and Rutgers University
}

\begin{abstract}
It is unprovable that every complete subalgebra of a countably closed complete Boolean algebra is countably closed.
\end{abstract}

Introduction. A partially ordered set $(P,<)$ is $\sigma$-closed if every countable chain in $P$ has a lower bound. A complete Boolean algebra $B$ is countably closed if $\left(B^{+},<\right)$has a dense subset that is $\sigma$-closed. In [2] the first author introduced a weaker condition for Boolean algebras, game-closed: the second player has a winning strategy in the infinite game where the two players play an infinite descending chain of nonzero elements, and the second player wins if the chain has a lower bound. In [1], Foreman proved that when $B$ has a dense subset of size $\aleph_{1}$ and is game-closed then $B$ is countably closed. (By Vojtáš [5] and Veličković [4] this holds for every $B$ that has a dense subset of size $2^{\aleph_{0}}$.) We show that, in general, it is unprovable that game-closed implies countably closed. We construct a model in which a $B$ exists that is game-closed but not countably closed. It remains open whether a counterexample exists in ZFC.

Being game-closed is a hereditary property: If $A$ is a complete subalgebra of a game-closed complete Boolean algebra $B$ then $A$ is game-closed. It is observed in [3] that every game-closed algebra is embedded in a countably closed algebra; in fact,

1991 Mathematics Subject Classification. 03E.

Key words and phrases. Boolean algebra, countably closed, game-closed, forcing.

The first author has been partially supported by the U.S.-Czechoslovakia cooperative grant INT-9016754 from the NSF.

The second author has been partially supported by the U.S.-Israel Binational Science Foundation. Publication number 565 
for a forcing notion $(P,<)$, being game-closed is equivalent to the existence of a $\sigma$ closed forcing $Q$ such that $P \times Q$ has a dense $\sigma$-closed subset. Hence the statement "every game-closed complete Boolean algebra is countably closed" is equivalent to the statement "every complete subalgebra of a countably closed complete Boolean algebra is countably closed".

Below we construct (by forcing) a model of $\mathrm{ZFC}+\mathrm{GCH}$ and in it a partial ordering $P$ of size $\aleph_{2}$ such that $B(P)$, the completion of $P$, is not countably closed, but $B(P \times C o l)$ is, where $C o l$ is the Lévy collapse of $\aleph_{2}$ to $\aleph_{1}$ (with countable conditions).

Theorem. It is consistent that there exists a partial ordering $(P,<)$ such that $B(P)$ is not countably closed but $B(P \times C o l)$ is countably closed.

\section{Forcing Conditions.}

We assume that the ground model satisfies $G C H$.

We want to construct, by forcing, a partially ordered set $\left(P,<_{P}\right)$ of size $\aleph_{2}$ that has the desired properties. We shall use as forcing conditions countable approximations of $P$. One part of a forcing condition will thus be a countable partial ordering $\left(A,<_{A}\right)$ with the intention that $A$ be a subset of $P$ and that the relation $<_{A}$ on $A$ be the restriction of $<_{P}$. As $P$ will have size $\aleph_{2}$, we let $P=\omega_{2}$, and so $A$ is a countable subset of $\omega_{2}$.

The second part of a forcing condition will be a countable set $B \subset A \times C o l$, a countable approximation of a dense set in the product ordering $P \times$ Col. The third part of a forcing condition will be a countable set $C$ of countable descending chains in $A$ that have no lower bound. Finally, a forcing condition includes a function that guarantees that the limit of the $B$ 's is $\sigma$-closed (and so $P \times C o l$ has a $\sigma$-closed dense subset).

Whenever we use < without a subscript, we mean the natural ordering of ordinal numbers.

Definition. For any set $X, \operatorname{Col}(X)$ is the set of all countable functions $q$ such 
Definition. The set $R$ of forcing conditions $r$ consists of quadruples $r=\left(\left(A_{r},<_{r}\right)\right.$, $\left.B_{r}, C_{r}, F_{r}\right)$ such that

(1) $A_{r}$ is a countable subset of $\omega_{2}$,

(2) $\left(A_{r},<_{r}\right)$ is a partially ordered set,

(3) if $b<_{r} a$ then $a<b$,

(4) $B_{r}$ is a countable subset of $A_{r} \times \operatorname{Col}\left(A_{r}\right)$, and for every $(p, q) \in B_{r}$, $p \in \operatorname{range}(q)$,

(5) $C_{r}$ is a countable set of countable sequences $\left\{a_{n}\right\}_{n=0}^{\infty}$ in $A_{r}$ with the property that $a_{0}>_{r} a_{1}>_{r} \cdots>_{r} a_{n}>_{r} \cdots$ and that $\left\{a_{n}\right\}_{n}$ has no lower bound in $A_{r}$,

(6) $F_{r}$ is a function of two variables, $\left\{a_{n}\right\}_{n} \in C_{r}$ and $(p, q) \in B_{r}$ such that $p \geq$ $a_{0}$, and range $\left(F_{r}\right) \subset \omega$. If $m=F_{r}\left(\left\{a_{n}\right\}_{n},(p, q)\right)$ then for every $\left(p^{\prime}, q^{\prime}\right) \in B_{r}$ stronger than $(p, q)$,

$$
\text { if } p^{\prime}<_{r} a_{m} \text { then } p^{\prime} \perp_{r}\left\{a_{n}\right\}_{n} \text { (i.e. } p^{\prime} \perp_{r} a_{k} \text { for some } k \text { ). }
$$

If $r, s \in R$ then $r<_{R} s$ ( $r$ is stronger than $s$ ) if

(7) $A_{r} \supseteq A_{s}$,

(8) $<_{r}$ and $<_{s}$ agree on $A_{s}$, and $\perp_{r}$ and $\perp_{s}$ agree on $A_{s}$; i.e. if $a, b \in A_{s}$ then $a<_{r} b$ iff $a<_{s} b$ and $a \perp_{r} b$ iff $a \perp_{s} b$ for all $\left.a, b \in A_{s}\right]$,

(9) $B_{r} \supseteq B_{s}$

(10) $C_{r} \supseteq C_{s}$,

(11) $F_{r} \supseteq F_{s}$.

The relation $<_{A}$ on $R$ is a partial ordering. We shall prove that the forcing extension by $R$ contains a desired example $\left(P,<_{P}\right)$. That $R$ is a cardinal-preserving model of $Z F C+G C H$ follows from the next two lemmas:

Lemma 1. $R$ is $\sigma$-closed.

Proof. Let $\left\{r_{n}\right\}_{n}$ be a sequence of conditions such that $r_{0}>_{R} r_{1}>_{R} \cdots>_{R} r_{n}>_{R}$ 
Assuming that for each $n, r_{n}=\left(\left(A_{n},<_{n}\right), B_{n}, C_{n}, F_{n}\right)$, we let $A_{r}=\bigcup_{n=0}^{\infty} A_{n}$, $B_{r}=\bigcup_{n=0}^{\infty} B_{n}, C_{r}=\bigcup_{n=0}^{\infty} C_{n}, F_{r}=\bigcup_{n=0}^{\infty} F_{n}$ and $<_{r}=\bigcup_{n=0}^{\infty}<_{n}$; we claim that $r=\left(\left(A_{r},<_{r}\right), B_{r}, C_{r}, F_{r}\right)$ is a condition, and is stronger than each $r_{n}$.

The triple $r$ has clearly properties (1)-(4). It is also easy to see that for every $n,<_{r}$ agrees with $<_{n}$ and $\perp_{r}$ agrees with $\perp_{n}$ on $A_{n}$. To verify (5), let $\left\{a_{n}\right\}_{n} \in C_{r}$. There is an $m$ such that $\left\{a_{n}\right\}_{n} \in C_{k}$ for all $k \geq m$, and therefore $\left\{a_{n}\right\}_{n}$ has no lower bound in any $A_{k}$. Thus $\left\{a_{n}\right\}_{n}$ has no lower bound in $A_{r}$. Finally, to verify $(6)$, let $F_{r}(\vec{a},(p, q))=m$ and let $\left(p^{\prime}, q^{\prime}\right)$ be stronger than $(p, q)$. Since $\left(^{*}\right)$ holds in $r_{n}$ where $n$ is large enough so that $\vec{a} \in C_{n}$ and $(p, q),\left(p^{\prime}, q^{\prime}\right) \in B_{n},\left({ }^{*}\right)$ holds in $r$ as well.

Therefore $r$ is a condition and for every $n, r$ is stronger than $r_{n}$.

Lemma 2. $R$ has the $\aleph_{2}$-chain condition.

Proof. If $W$ is a set of conditions of size $\aleph_{2}$, then a $\Delta$-system argument (using $\mathrm{CH})$ yields two conditions $r, s \in W$ such that if $r=\left(\left(A_{r},<_{r}\right), B_{r}, C_{r}, F_{r}\right)$ and $s=\left(\left(A_{s},<_{s}\right), B_{s}, C_{s}, F_{s}\right)$, then there is a $D$ (the root of the $\Delta$-system) such that $D=A_{r} \cap A_{s}, \sup D<\min \left(A_{r}-D\right), \sup A_{r}<\min \left(A_{s}-D\right),<_{r}$ and $<_{s}$ agree on $D, \perp_{r}$ and $\perp_{s}$ agree on $D, B_{r} \cap(D \times \operatorname{Col}(D))=B_{s} \cap(D \times \operatorname{Col}(D))$, $C_{r} \cap D^{\omega}=C_{s} \cap D^{\omega}$, and $F_{r}(\vec{a},(p, q))=F_{s}(\vec{a},(p, q))$ whenever $\vec{a} \in C_{r} \cap D^{\omega}$ and $(p, q) \in B_{r} \cap(D \times \operatorname{Col}(D))$.

Moreover, there exists a mapping $\pi$ of $A_{s}$ onto $A_{r}$ that is an isomorphism between $s$ and $r$ and is the identity on $D$.

Let $t=\left(\left(A_{t},<_{t}\right), B_{t}, C_{t}, F_{t}\right)$ where $A_{t}=A_{r} \cup A_{s}, B_{t}=B_{r} \cup B_{s}, C_{t}=C_{r} \cup C_{s}$, $<_{t}=<_{r} \cup<_{s}$, and $F_{t}$ will be defined below such that $F_{t} \supseteq F_{r} \cup F_{s}$. We claim that $t$ is a condition, and is stronger than both $r$ and $s$; thus $r$ and $s$ are compatible. Properties (1)-(4) are easy to verify. It is also easy to see that $<_{t}$ agrees with $<_{r}$ on $A_{r}$ and with $<_{s}$ on $A_{s}$, and $\perp_{t}$ agrees with $\perp_{r}$ on $A_{r}$ and with $\perp_{s}$ on $A_{s}$.

Note that if $a \in A_{r}-D$ and $b \in A_{s}-D$ then $a \perp_{t} b$. Thus if $\left\{a_{n}\right\}_{n}$ is in $C_{r}$ but not in $C_{s}$ (or vice versa) then $\left\{a_{n}\right\}_{n}$ has no lower bound in $A_{r} \cup A_{s}$, and so (5) 
In order to deal with (6), we first verify it for the values of $F_{t}$ inherited from either $r$ or $s$. Thus let $\vec{a} \in C_{r},(p, q) \in B_{r}, m=F_{r}(\vec{a},(p, q))$ and let $\left(p^{\prime}, q^{\prime}\right) \in B_{t}$ be stronger than $(p, q)$. (The argument for $s$ in place of $r$ is completely analogous.) If $\left(p^{\prime}, q^{\prime}\right) \in B_{r}$ then $(*)$ holds in $r$ and therefore in $t$. Thus assume that $\left(p^{\prime}, q^{\prime}\right) \in B_{s}$.

Since $p^{\prime} \in A_{s}$ and $p^{\prime}<_{t} p$, it follows that $p \in D$, and since $\operatorname{range}(q) \subseteq \operatorname{range}\left(q^{\prime}\right) \subseteq$ $A_{s}$, we have $(p, q) \in B_{s}$. Now if $\vec{a} \in C_{s}$ then $F_{s}(\vec{a},(p, q))=F_{r}(\vec{a},(p, q))$ and so $p^{\prime}$ satisfies $\left(^{*}\right)$ in $s$ and hence in $t$. If $\vec{a} \notin C_{s}$ and $p^{\prime} \notin A_{r}$ then $p^{\prime} \perp_{t} \vec{a}$ and again $p^{\prime}$ satisfies $(*)$.

The remaining case is when $p^{\prime} \in D$ and $(p, q) \in B_{r} \cap B_{s}$. Since $\left(p^{\prime}, \pi q^{\prime}\right)=$ $\left(\pi p^{\prime}, \pi q^{\prime}\right)$ is stronger than $(p, q)=(\pi p, \pi q), p^{\prime}$ satisfies $\left(^{*}\right)$ in $r$ and therefore in $t$.

To complete the verification of (6) we define $F_{t}(\vec{a},(p, q))$ for those $\vec{a}$ and $(p, q)$ that come from the two different conditions. Let $\vec{a} \in C_{r}-C_{s}$ and $(p, q) \in B_{s}-B_{r}$ (the other case being analogous) be such that $p \geq a_{0}$. We let $F_{t}(\vec{a},(p, q))$ be the least $m$ such that $a_{m} \notin D$.

Let $\left(p^{\prime}, q^{\prime}\right) \in B_{t}$ be stronger than $(p, q)$; we'll show that $p^{\prime} \nless_{t} a_{m}$. This is clear if $p^{\prime} \in D$. If $p^{\prime} \notin D$ then $p^{\prime}$ cannot be in $A_{r}$ because by (4) $p^{\prime} \in \operatorname{range}\left(q^{\prime}\right) \subseteq$ range $(q) \subseteq A_{s}$. It follows that $p^{\prime} \perp_{t} a_{m}$.

Therefore $t$ is a condition and is stronger than both $r$ and $s$.

Let $G$ be a generic filter on $R$. In $V_{G}$, we let $P=\bigcup\left\{A_{r}: r \in G\right\},<_{P}=\bigcup\left\{<_{r}\right.$ : $r \in G\}$, and $Q=\bigcup\left\{B_{r}: r \in G\right\} .\left(P,<_{P}\right)$ is a partial ordering and $Q \subset P \times C o l$. We shall prove that $Q$ is $\sigma$-closed and is dense in $P \times C o l$, and that the complete Boolean algebra $B(P)$ does not have a dense $\sigma$-closed subset.

Lemma 3. $P=\omega_{2}$.

Proof. We prove that for every $s$ and every $p \in \omega_{2}$ there exists an $r<_{R} s$ such that $p \in A_{r}$. But this is straightforward: let $A_{r}=A_{s} \cup\{p\}, B_{r}=B_{s}, C_{r}=C_{s}$, $F_{r}=F_{s}$ and $<_{r}=<_{s}$; properties (1)-(11) are easily verified. (Note that $p \perp_{r}$ a for all $\left.a \in A_{s}.\right)$ 
Proof. Let $s$ be a condition and let $p_{0} \in A_{s}$ and $q_{0} \in C o l$. We shall find an $r<_{R} s$, $p \in A_{r}$ and $q \supset q_{0}$ such that $p<_{r} p_{0}$ and $(p, q) \in B_{r}$ : Let $p$ be an ordinal greater than $p_{0}$ and $p \notin A_{s}$, let $q \in C o l$ be such that $q \supset q_{0}$ and $p \in$ range $(q)$, and let $A_{r}=A_{s} \cup$ range $(q), B_{r}=B_{s} \cup\{(p, q)\}, C_{r}=C_{s}$, and let $<_{r}$ be the partial order of $A_{r}$ that extends $<_{s}$ by making $p<_{r} p_{0}$. Finally, let $F_{r}(\vec{a},(p, q))=0$ for all $\vec{a} \in C_{r}$.

To see that $r=\left(\left(A_{r},<_{r}\right), B_{r}, C_{r}, F_{r}\right)$ is a condition, note that for every $\vec{a} \in C_{r}$, $p$ is not a lower bound of $\vec{a}$ (because $p_{0}$ isn't) and hence $p \perp_{r} \vec{a}$. This implies both (5) and (6). Since adding $p$ does not effect the relation $\perp$ on $A_{s}$, we have (8) and so $r$ is stronger than $s$.

Next we prove that $Q$ is $\sigma$-closed.

Lemma 5. If $u=\left\{\left(p_{n}, q_{n}\right)\right\}_{n=0}^{\infty}$ is a descending chain in $Q$ then $u$ has a lower bound.

Proof. Let $\dot{u}$ be a name for a descending chain and let $s$ be a condition. By extending $s \omega$ times if necessary ( $R$ is $\sigma$-closed), we may assume that there is a sequence $u=\left\{\left(p_{n}, q_{n}\right)\right\}_{n=0}^{\infty}$ in $\omega_{2} \times C o l$ such that $s$ forces $\dot{u}=u$, such that for every $n, p_{n} \in A_{s},\left(p_{n}, q_{n}\right) \in B_{s}$, that $p_{0}>_{s} p_{1}>_{s} \cdots>_{s} p_{n}>\cdots$ is a descending chain in $\left(A_{s},<_{s}\right)$ and that $q_{0} \subset q_{1} \subset \cdots \subset q_{n} \subset \ldots$

Let $p$ be an ordinal greater than $\sup A_{s}$, let $q \supseteq \bigcup_{n=0}^{\infty} q_{n}$ be such that $p \in$ $\operatorname{range}(q) \subseteq A_{s} \cup\{p\}$, let $A_{r}=A_{s} \cup\{p\}, B_{r}=B_{s} \cup\{(p, q)\}, C_{r}=C_{s}$, and let $<_{r}$ be the partial order of $A_{r}$ that extends $<_{s}$ by making $p$ a lower bound of $\left\{p_{n}\right\}_{n=0}^{\infty}$. Finally, let $F_{r}(\vec{a},(p, q))=0$ for all $\vec{a} \in C_{r}$ and $r=\left(\left(A_{r},<_{r}\right), B_{r}, C_{r}, F_{r}\right)$.

We shall show that for every $\vec{a} \in C_{s}, p$ is not a lower bound of $\vec{a}$. This implies that $p \perp_{r} \vec{a}$ and (5) and (6) follow. Since making $p$ a lower bound of $\left\{p_{n}\right\}_{n}$ does not effect the relation $\perp$ on $A_{s}$, we'll have (8) and hence $r<_{R} s$. In $r,(p, q)$ is a lower bound of $u$.

Thus let $\vec{a}=\left\{a_{k}\right\}_{k} \in C_{s}$. We claim that 
If $p_{n}<a_{0}$ for all $n$ then we let $k=0$ because then $a_{0} \nless_{s} p_{n}$ for all $n$.

Otherwise let $N$ be the least $N$ such that $p_{N} \geq a_{0}$, and let $m=F_{s}\left(\vec{a},\left(p_{N}, q_{N}\right)\right)$. Either $a_{m} \nless_{s} p_{n}$ for all $n$ and we are done (with $k=m$ ) or else $a_{m}<_{s} p_{M}$ for some $M \geq N$. By $\left(^{*}\right)$ there exists some $k$ such that $p_{M} \perp_{s} a_{k}$ and hence $a_{k} \nless_{s} p_{n}$ for all $n$.

Finally, we shall prove that $B(P)$ is not countably closed.

Lemma 6. The complete Boolean algebra $B(P)$ does not have a dense $\sigma$-closed subset.

Proof. Assume that $B(P)$ does have a dense $\sigma$-closed subset $D$. For $a, b \in P$, we define

$$
a \prec b \quad \text { if } \quad a<_{P} b \quad \text { and } \quad \exists d \in D \quad \text { such that } \quad a<_{B} d<_{B} b \text {. }
$$

The relation $\prec$ is a partial ordering of $P,(P, \prec)$ is $\sigma$-closed, $a \prec b$ implies $a<_{P} b$ and for every $a \in P$ there is some $b \in P$ such that $b \prec a$.

Toward a contradiction, let $s$ be a condition and assume that $s$ forces the preceding statement. For each $\alpha<\omega_{2}$, there exist a condition $s_{\alpha}$ stronger than $s$, and a descending chain $\left\{c_{n}^{\alpha}\right\}_{n}$ in $A_{s_{\alpha}}$ such that $c_{0}^{\alpha} \geq \alpha$ and that for every $n$, $s_{\alpha} \Vdash c_{n+1}^{\alpha} \prec c_{n}^{\alpha}$.

By a $\Delta$-system argument we find among these a countable sequence $r_{n}=s_{\alpha_{n}}=$ $\left(\left(A_{n},<_{n}\right), B_{n}, C_{n}, F_{n}\right)$ and a set $D$ such that for every $m$ and $n$ wih $m<n$ we have $D=A_{m} \cap A_{n}, \sup D<\min \left(A_{m}-D\right), \sup A_{m}<\min \left(A_{n}-D\right),<_{m}$ and $<_{n}$ agree on $D, \perp_{m}$ and $\perp_{n}$ agree on $D, B_{m} \cap(D \times \operatorname{Col}(D))=B_{n} \cap(D \times \operatorname{Col}(D))$, $C_{m} \cap D^{\omega}=C_{n} \cap D^{\omega}$, and $F_{m}(\vec{a},(p, q))=F_{n}(\vec{a},(p, q))$ whenever $\vec{a} \in C_{m} \cap D^{\omega}$ and $(p, q) \in B_{m} \cap(D \times \operatorname{Col}(D))$. Moreover, there exists a mappings $\pi_{m n}$ of $A_{m}$ onto $A_{n}$ that is an isomorphism between $\left(r_{m},\left\{c_{k}^{\alpha_{m}}\right\}_{k}\right)$ and $\left(r_{n},\left\{c_{k}^{\alpha_{n}}\right\}_{k}\right)$ and is the identity on $D$. We also let $\pi_{n m}=\pi_{m n}{ }^{-1}, \pi_{m m}=i d$ and assume that the $\pi_{m n}$ form a commutative system. Note that for every $n$ and $k, c_{k}^{\alpha_{n}} \notin D$. 
sequence"

$$
u_{2 n}=a_{n}^{n}, \quad u_{2 n+1}=b_{n}^{n} .
$$

We shall find a condition $t=\left(\left(A_{t},<_{t}\right), B_{t}, C_{t}, F_{t}\right)$ stronger than all $r_{n}$ such that the diagonal sequence $\vec{u}$ is a descending chain and belongs to $C_{t}$. Since $t \Vdash b_{n}^{n} \prec a_{n}^{n}$ for every $n$, it forces that $(P, \prec)$ is not $\sigma$-closed. This will complete the proof.

To construct $t$ we first let $A_{t}=\bigcup_{n=0}^{\infty} A_{n}$ and $B_{t}=\bigcup_{n=0}^{\infty} B_{n}$. Let $<_{t}$ be the minimal partial ordering extending $\bigcup_{n=0}^{\infty}<_{n}$ such that for every $n, a_{n+1}^{n+1}<_{t} b_{n}^{n}$. Before proceeding to define $C_{t}$ and $F_{t}$ we shall prove some properties of $\left(A_{t},<_{t}\right)$.

Lemma 7. (i) Let $m<n$ and let $y \in A_{m}-D$ and $x \in A_{n}-D$. If $x<_{t} y$ then $x \leq_{n} a_{n}^{n}$ and $b_{m}^{m} \leq_{m} y$. If $x$ and $y$ are compatible in $<_{t}$ then $b_{m}^{m} \leq_{m} y$.

(ii) For all $m$ and $n$, if $x \in A_{n}$ and $y \in A_{m}$ and if $x<_{t} y$ then $x<_{n} \pi_{m n} y$ (and $\left.\pi_{n m} x<_{m} y\right)$. In particular, if $x, y \in A_{n}$ then $x<_{t} y$ if and only if $x<_{n} y$.

(iii) For all $m$ and $n$, if $x \in A_{n}$ and $y \in A_{m}$ and if $x$ and $y$ are compatible in $<_{t}$ then $x$ and $\pi_{m n} y$ are compatible in $<_{n}$ (and $\pi_{n m} x$ and $y$ are compatible in $\left.<_{m}\right)$. In particular, if $x, y \in A_{n}$ then $x \perp_{t} y$ if and only if $x \perp_{n} y$.

Proof. (i) The first statement is an obvious consequence of the definition of $<_{t}$, and the second follows because any $z$ such that $z \leq_{t} x$ is in some $A_{k}-D$ where $k \geq n$.

(ii) Let $x \in A_{n}$ and $y \in A_{m}$ and let $x<_{t} y$. First assume that $y \notin D$ (and so $x \notin D$.) Necessarily, $m \leq n$ and if $m=n$ then clearly $x<_{n} y$. Thus consider $m<n$. By (i) $x \leq_{n} a_{n}^{n}<_{n} b_{m}^{n}=\pi_{m n}\left(b_{m}^{m}\right) \leq_{n} \pi_{m n} y$.

Now assume that $y \in D$ and proceed by induction on $x$. If $x \in D$ then $x<_{n} y$. If $x \notin D$ then either $x<_{n} y$ or there exists some $z \notin D$ such that $x<_{t} z<_{t} y$, and by the induction hypothesis $z<_{k} \pi_{m k} y$ (where $z \in A_{k}$ ). Applying the preceding paragraph to $x$ and $z$ we get $\pi_{n k} x<_{k} z$ and hence $\pi_{n k} x<_{k} \pi_{m k} y$. The statement now follows.

(iii) Let $x \in A_{n}$ and $y \in A_{m}$ and let $z \in A_{k}$ be such that $z<_{t} x$ and $z<_{t} y$. By (ii) we have $\pi_{k n} z<_{n} x$ and $\pi_{k m} z<_{m} y$. Hence $\pi_{k n} z=\pi_{m n} \pi_{k m} z<_{n} \pi_{m n} y$. The 
Lemma 7 guarantees that $t$ will be stronger than every $r_{n}$. Another consequence is that if $\vec{a} \in C_{n}$ then $\vec{a}$ has no lower bound in $<_{t}$ : if $x \in A_{m}$ were a lower bound then $\pi_{m n} x$ would be a lower bound in $<_{n}$.

Let $C_{t}=\bigcup_{n=0}^{\infty} C_{n} \cup\{\vec{u}\}$. Every sequence in $C_{t}$ is a descending chain in $<_{t}$ without a lower bound (clearly, $\vec{u}$ has no lower bound).

Lemma 8. For all $k$ and $n$, if $(p, q) \in B_{k}-B_{n}$ and if $\left(p^{\prime}, q^{\prime}\right) \in B_{t}$ is stronger than $(p, q)$ then $\left(p^{\prime}, q^{\prime}\right) \in B_{k}-B_{n}$.

Proof. Since $(p, q) \notin B_{n}$, we have either $\operatorname{range}(q) \nsubseteq D$ or $p \notin D$, in which case $p \in \operatorname{range}(q)$ by $(4)$ and again range $(q) \nsubseteq D$. Since $q \subseteq q^{\prime}$ it must be the case that $\left(p^{\prime}, q^{\prime}\right) \in B_{k}-B_{n}$.

We shall now define $F_{t}$ so that $F_{t} \supset \bigcup_{n=0}^{\infty} F_{n}$ and verify (6). This will complete the proof.

First we let $F_{t}(\vec{a},(p, q))=F_{n}(\vec{a},(p, q))$ whenever the right-hand side is defined; we have to show that (6) holds in $t$. Let $m=F_{n}(\vec{a},(p, q))$ and let $\left(p^{\prime}, q^{\prime}\right) \in B_{k}$ be stronger than $(p, q)$. It follows from Lemma 8 that $(p, q) \in B_{k}$. Now $\left(\pi_{k n} p^{\prime}, \pi_{k n} q^{\prime}\right)$ is stronger than $\left(\pi_{k n} p, \pi_{k n} q\right)=(p, q)$ and $(*)$ holds for $\pi_{k n} p^{\prime}$ in $r_{n}$. If $p^{\prime}<_{t} a_{m}$ then by Lemma $7 \pi_{k n} p^{\prime}<_{n} a_{m}$ and hence $\pi_{k n} p^{\prime} \perp_{n} \vec{a}$. By Lemma 7 again, $p^{\prime} \perp_{t} \vec{a}$.

Next, let $\vec{a}$ and $(p, q)$ be such that $\vec{a} \in C_{n}-C_{k},(p, q) \in B_{k}-B_{n}$ and $p \geq a_{0}$. If $k<n$, we have $\pi_{k n} p \geq p \geq a_{0}$ and we let $F_{t}(\vec{a},(p, q))=F_{n}\left(\vec{a},\left(\pi_{k n} p, \pi_{k n} q\right)\right)$. To verify $(6)$, let $m=F_{t}(\vec{a},(p, q))$ and let $\left(p^{\prime}, q^{\prime}\right) \in B_{t}$ be stronger than $(p, q)$. By Lemma $8\left(p^{\prime}, q^{\prime}\right) \in B_{k}$, and $\left(\pi_{k n} p^{\prime}, \pi_{k n} q^{\prime}\right)$ is stronger (in $\left.r_{n}\right)$ than $\left(\pi_{k n} p, \pi_{k n} q\right)$. If $p^{\prime}<_{t} a_{m}$ then by Lemma $7 \pi_{k n} p^{\prime}<_{n} a_{m}$ and so $\pi_{k n} p^{\prime} \perp_{n} \vec{a}$. By Lemma 7 again, $p^{\prime} \perp_{t} \vec{a}$.

If $k>n$, we let $F_{t}(\vec{a},(p, q))$ be the least $m$ such that $a_{m} \notin D$ and that $b_{n}^{n} \not \leq a_{m}$. To verify $(6)$, let $\left(p^{\prime}, q^{\prime}\right) \in B_{t}$ be stronger than $(p, q)$. If $p^{\prime} \in D$ then $p^{\prime} \nless_{t} a_{m}$ and if $p^{\prime} \notin D$ then by Lemma $7(\mathrm{i}) p^{\prime} \perp_{t} a_{m}$. In either case, (6) is satisfied.

Finally, we define $F_{t}(\vec{u},(p, q))$. Thus let $(p, q) \in B_{t}$ be such that $p \geq u_{0}$. Since $u_{0}=a_{0}^{0} \notin D$, we have $p \notin D$. Let $n$ be the $n$ such that $p \in A_{n}$. We let $F_{t}(\vec{u},(p, q))=$ 
stronger than $(p, q)$. Since $p \in A_{n}-D$, by Lemma 8 we have $\left(p^{\prime}, q^{\prime}\right) \in B_{n}$ and therefore $p^{\prime} \in A_{n}-D$. But $a_{n+1}^{n+1} \in A_{n+1}-D$ and so $p^{\prime} \nless_{t} a_{n+1}^{n+1}$. Therefore (6) holds.

\section{REFERENCES}

1. M. Foreman, Games played on Boolean algebras, J. Symbolic Logic 48 (1983), 714-723.

2. T. Jech, A game-theoretic property of Boolean algebras, in: Logic Colloquium 77 (A. Macintyre et al., eds.), North-Holland Publ. Co., Amsterdam 1978, pp. 135-144.

3. T. Jech, More game-theoretic properties of Boolean algebras, Annals of Pure and Applied Logic 26 (1984), 11-29.

4. B. Veličković, Playful Boolean algebras, Transactions of the American Math. Society 296 (1986), $727-740$.

5. P. Vojtáš, Game properties of Boolean algebras, Comment. Math. Univ. Carol. 24 (1983), 349-369.

Department of Mathematics, The Pennsylvania State University, University Park, PA 16803, USA

Institute of Mathematics, The Hebrew University, Jerusalem, Israel, and Department of Mathematics, Rutgers University, New Brunswick, NJ 08903, USA

E-mail address: jech@math.psu.edu, shelah@sunrise.huji.ac.il, shelah@math.rutgers.edu 